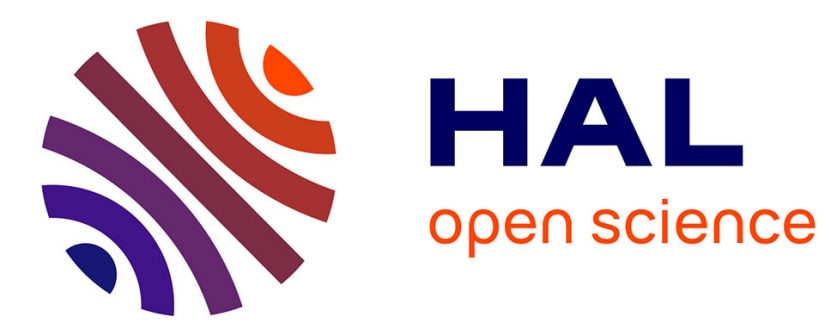

\title{
Body-color plasticity of the English grain aphid in response to light in both laboratory and field conditions
}

Kévin Tougeron, Joan van Baaren, Jennifer Town, Dana Nordin, Tim Dumonceaux, Tyler Wist

\section{- To cite this version:}

Kévin Tougeron, Joan van Baaren, Jennifer Town, Dana Nordin, Tim Dumonceaux, et al.. Bodycolor plasticity of the English grain aphid in response to light in both laboratory and field conditions. Evolutionary Ecology, 2021, 35 (1), pp.147-162. 10.1007/s10682-020-10088-4 . hal-03034160

\section{HAL Id: hal-03034160 https://hal.science/hal-03034160}

Submitted on 18 Dec 2020

HAL is a multi-disciplinary open access archive for the deposit and dissemination of scientific research documents, whether they are published or not. The documents may come from teaching and research institutions in France or abroad, or from public or private research centers.
L'archive ouverte pluridisciplinaire HAL, est destinée au dépôt et à la diffusion de documents scientifiques de niveau recherche, publiés ou non, émanant des établissements d'enseignement et de recherche français ou étrangers, des laboratoires publics ou privés. 


\section{Body-color plasticity of the English grain aphid in response to light in both laboratory and field conditions}

Tougeron K. ${ }^{1,2}$, van Baaren J. ${ }^{1}$, Town J. ${ }^{3}$, Nordin D. ${ }^{3}$, Dumonceaux T. ${ }^{3}$ \& Wist T. ${ }^{3}$

Corresponding author: kevin.tougeron@uclouvain.be

ORCID: Kévin Tougeron 0000-0003-4897-3787 / Joan van Baaren 0000-0002-8552-9645 / Jennifer Town 0000-0002-9054-2369 / Tim Dumonceaux 0000-0001-5165-0343 / Tyler Wist 0000-0003-38202487

${ }^{1}$ UMR CNRS 6553 Ecobio, Université de Rennes 1, 263 avenue du général Leclerc, 35042, Rennes, France.

${ }^{2}$ Earth and Life Institute, Biodiversity Research Centre, Université catholique de Louvain, 1348 Louvain-la-Neuve, Belgium

${ }^{3}$ Agriculture and Agri-Food Canada, Government of Canada, Saskatoon Research and Development Centre, Saskatoon, SK, Canada, S7N 0X2

CRediT statement: Conceptualization and Methodology: Kévin Tougeron, Joan van Baaren, Jennifer Town, Dana Nordin, Tim Dumonceaux and Tyler Wist; Formal analysis: Kévin Tougeron, Jennifer Town and Tyler Wist; Writing: Kévin Tougeron; Funding acquisition: Joan van Baaren, Tim Dumonceaux and Tyler Wist; Supervision: Joan van Baaren, Tim Dumonceaux and Tyler Wist

\section{Abstract}

The occurrence of different color patterns in a population of a species can depend on genetic variations or plasticity to environmental conditions. Body color variation is under selection because it is involved in several ecological processes such as camouflage for prey-predator interactions or resistance to environmental variations. Among insects, aphids are known to produce different body-color morphs depending on their biotic and abiotic environments and their bacterial endosymbionts. The Englishgrain aphid (EGA) Sitobion avenae produces both red and green morphs in cereal fields. Using both field studies on the Canadian prairies (Saskatchewan) and laboratory experiments, we aimed to understand the mechanisms that triggers plasticity in body coloration to better understand the ecological role of body coloration and color-change evolved by animals, including aphids. We first analyzed green and red morph EGA distribution on wheat ears in different fields and showed that red aphids were mostly located at the top of the ear and green aphids at the bottom. Then, using DNA sequencing, we showed that red and green morphs did not strongly differ in their bacterial endosymbiont composition and abundances. Finally, using a climate-chamber setup in the laboratory, we highlighted that EGA body-coloration is under light-intensity control and that it is possible to turn aphids from green back to red within a few days, and from red back to green within a couple of weeks (low-to-high and high-to-low light intensities, respectively). Light-intensity-controlled color-change likely results in adaptive plasticity in response to shifts in environmental conditions that can occur over the lifespan of an aphid, and is fully reversible, even at the adult stage.

Keywords: Behavioral ecology; Endosymbiont; Light-intensity; Plasticity; Polymorphism; Polyphenism; Sitobion avenae; Wheat 


\section{Introduction}

Many animals have evolved the capacity to produce a range of color patterns at the intraspecific level. Body color variation in the population of a species plays major roles in the animal kingdom and is involved in several ecological processes such as mate finding, thermal tolerance, food foraging, social interactions and camouflage (Duarte et al., 2017; Ford, 1966; Majerus, 1998; Stevens and Merilaita, 2009). Body-color is thus central to evolutionary ecology because it strongly influences individual fitness in a given environment (Abram et al., 2015; Forsman et al., 2008). Color variation can be due to genetic polymorphism within a population, but can also arise from phenotypic plasticity (polyphenism), in which case body-color varies across different temporal scales depending on both biotic and abiotic environmental cues (Cloudsley-Thompson, 1999; Cott, 1940; Hazel, 2002; Tanaka et al., 2016). Body-color polyphenism in the American grasshopper Schistocerca americana is, for instance, influenced by developmental temperature, with more reddish and darker forms observed at lower temperatures (Tanaka, 2004). The surge damselfish Chrysiptera leucopoma expresses two bodycolor morphs that are set up during ontogeny depending on habitat background color, and that are reversible, although only before the adult stage (Frédérich et al., 2010). However, such examples of reversible color change within individuals are quite rare and unravelling the mechanistic bases of such changes is important.

Among insects, aphids (Hemiptera: Aphididae) are interesting models to study adaptive phenotypic variations because they produce different types of morphologically distinct individuals (i.e., morphs) that coexist spatially and temporally (Hille Ris Lambers, 1966; Dixon, 1985; Loxdale and Lushai, 2003; Hougardy and Mills, 2008). The onset of a specific morph serves different ecological purposes and is triggered by different environmental factors. For instance winged aphids are produced to ensure dispersion in case of overcrowding on the plant (Sutherland, 1969) or escaping predators (Dixon and Agarwala, 1999), while sexual morphs are produced when temperature and photoperiod decrease, and lead to the production of eggs that will overwinter (Hand and Wratten, 1985). Color variation has been well studied in aphids, especially in the pea aphid, Acyrthosiphon pisum, which displays pink and green morphs (e.g., Caillaud and Losey, 2010), but less in other species such as the English-grain aphid (EGA), Sitobion avenae, which displays a panel of colors transitioning between green and red morphs (brown, chestnut, pink, etc.) (Weber, 1985; Jenkins et al., 1999). Coloration results from variation in pigment types and abundances in the tegument or the hemolymph (Bowie et al., 1966; Jenkins et al., 1999; Kayser, 1982; Sullivan, 2008). In S. avenae, apterous red morphs have a shorter longevity but a higher fecundity than green morphs, suggesting physiological differences between color morphs, but also that they could succeed differently in dissimilar environments (Araya et al., 1996).

The production of different color morphs may vary following the aphid clonal lineages which highlights the genetically-based variation of body colors (Moran and Jarvik, 2010). For instance in the pea aphid in which two morphs (pink and green) are produced, color polymorphism is determined by a single biallelic locus, pink being dominant to green (Caillaud and Losey, 2010). Phylogenetic analyses have shown that these aphid genes are derived from fungal genes (Moran and Jarvik, 2010). Color polymorphism in aphids is maintained by different mechanisms, depending on the species; it is for example under balanced, density-dependent selection in the field, either by mutualistic relationships (for example with ants, Watanabe et al., 2016), or by predation and parasitism (Losey et al., 1997). Color indeed plays a major role in trophic interactions since a lot of predator insect species rely although not exclusively - on visual cues to detect their prey, which has in turn led to the evolution of 
camouflage strategies (Harmon et al., 1998; Théry and Gomez, 2010). In the pea aphid, green morphs are more susceptible to predation by ladybugs than pink morphs which are more susceptible to parasitoids (Losey et al., 1997; Libbrecht et al., 2007). Pink morphs are more likely to drop off a plant than green morphs when attacked by a predator as a defensive behavior, but less likely to drop off the plant in the presence of parasitoids (Braendle and Weisser, 2001; Dion et al., 2011). While such fieldbased studies have not been conducted on other aphid species, morph differences regarding sensitivity to parasitism have been reported in S. avenae. For example, the attack behavior of the aphid parasitoid Aphidius rhopalosiphi did not differ between S. avenae color morphs, nor did the aphid defense behavior, but Ankersmit et al. (1981) noted that red morphs have better physiological resistance to parasite development.

However, there can be color variation within aphid clones (i.e., among individuals sharing the same genotype) and within individuals that can be induced by plasticity to environmental factors such as temperature, photoperiod, diet, host-plant and bacterial infection (Hille Ris Lambers, 1966; Markkula and Rautapää, 1967; Shu-Sheng and Carver, 1982; Alkhedir et al., 2010; Tsuchida et al., 2010). In this case, coloration is the result of phenotypic plasticity and may thus be reversed if the given environmental factor reverts to the previous state. For example, caterpillars of the geometrid peppered moth (Biston betularia) not only match closely the color of their host plant, but also have the potential to change color until the final larval instar if the substrate changes (Noor et al., 2008). In some aphid species, color patterns appear to be strongly linked to the presence of bacterial endosymbionts such as the obligate symbionts Buchnera aphidicola or facultative symbionts such as Hamiltonella defensa, Serratia symbiotica or Rickettsiella sp. that can provide pigment precursors that animals, including aphids, are unable to synthetize on their own (Douglas, 1998). For instance, Rickettsiella symbionts that infect the pea aphid result in an aphid color change from pink to green (Tsuchida et al., 2010). In S. avenae, it is unknown whether endosymbiotic bacteria are involved in the body coloration process.

Having the capacity to change body-color is extremely interesting in an eco-evo perspective, however, there is still a lack of knowledge on ecological mechanisms that lead to body color-changes through plasticity in most animal species. As explained before, $S$. avenae does not express a strict pink-green polymorphism as $A$. pisum does, but rather a color gradient from green to red depending on carotenoid abundance in the hemolymph of different morphs (Alkhedir et al., 2010; Jenkins et al., 1999), which might reflect the plasticity of this trait. Eight types of carotenoids have been found in the hemolymph of brown-red morph compared to one in green morph S. avenae (Jenkins et al. 1999). Changes in lightintensity and photoperiod governs a lot of insects' phenotypic variations across different time-scales (Saunders, 2012), and might also be responsible for body-color adaptive plasticity, as reported in other animals (Lin et al., 2009; Lymbery, 1992; Stegen et al., 2004; Tanaka et al., 2016). Markkula and Rautapää (1967) showed changes in the body coloration of the aphid Macrosiphum euphorbiae related to temperature, photoperiod and light-intensity, underlying seasonal changes in body coloration in the field. In some aphid species, such as the sycamore aphid, specific color may absorb solar radiation which would be advantageous in cool weather (Dixon, 1972). The stink bug Podisus maculiventris can lay eggs ranging from unpigmented to heavily pigmented UV-protected eggs, which is the result of females actively controlling for egg pigmentation depending on characteristics of the laying surface (Abram et al., 2015). Other examples of adaptive responses to light exposure are known, such as Chinese longsnout catfishes that have darker skins under high light intensities, probably as a way to reduce stress (Han et al., 2005). Light-intensity thus seems to be a critical factor involved in the plastic 
control of body coloration in some animal species, including aphid species. Therefore, we hypothesized that color polyphenism of EGA in the field is due to aphid exposure to different light intensities.

In this study, we first aimed to assess the levels of different morphs of aphids in the fields and their repartition on the wheat plant. We expected different color morphs to occupy different locations on the plant, either as a behavioral adaptation or as a direct response to different light intensities. Then, we wanted to understand if coloration was due to differential presence of bacterial endosymbionts. We expected bacterial communities to differ between red and green morphs, as it is already known in other aphid species. Finally, we performed lab experiments to test the effect of different light intensity exposure on EGA body-color and assessed the reversibility of this polyphenism. We expected change in light exposure to trigger plasticity in body-color of aphids, and color-change to be reversible within a generation.

\section{Material and Methods}

Field study

We surveyed five different wheat fields in the province of Saskatchewan (Canada): a commercial field near Nipawin $\left(53.40^{\circ} \mathrm{N}, 104.56^{\circ} \mathrm{W}\right)$, University of Saskatchewan's Kernen Research Farm $\left(52.16^{\circ} \mathrm{N}\right.$, $\left.106.55^{\circ} \mathrm{W}\right)$, Agriculture and Agri-Food Canada's Melfort Research Farm $\left(52.82^{\circ} \mathrm{N}, 104.60^{\circ} \mathrm{W}\right)$, a commercial field near Fairy Glen $\left(53.04^{\circ} \mathrm{N}, 104.52^{\circ} \mathrm{W}\right)$ and a commercial field near Wakaw $\left(52.63^{\circ} \mathrm{W}\right.$, $105.75^{\circ} \mathrm{N}$ ) in summer 2015 . All fields were conventionally managed. Each field was visited one time and data was collected between 10 and 26 August, when wheat ears had partially dried and harvest was underway. In each field, the first wheat plants containing at least one aphid of each morph were selected. Aphids were counted on a total of 65 wheat ears of at least $10 \mathrm{~cm}$ length (from 4 to 15 wheat ears depending on the fields). In most cases, both EGA morphs were found on each wheat ear when scouting the fields. Green and red morphs of Sitobion avenae (Figure 1) were counted on different locations of the wheat ears using a break of $1 \mathrm{~cm}$ from bottom to top of the ears. First larval instar were excluded from the count because they may not get their definitive color at this point (Tsuchida et al., 2010). It is important to notice that differences in repartition patterns between morphs can only be assessed as relative proportions, because some wheat heads had more aphids of one color type than of the other.

Data was divided into "bottom" ( $\mathrm{cm} 1$ to 6 ) and "top" ( $\mathrm{cm} 7$ to top of the wheat ear) locations, which gives an almost equal repartition of the categories on the wheat ear. Morph repartition data was analyzed by fitting a Generalized Linear Mixed Model (GLMM) with Poisson distribution to the data (Bates et al., 2018) using the number of aphids as the response variable, the location on the wheat ear (bottom or top) and the morph and the interaction factor as explanatory variables, and the wheat field as a random effect. Distribution assumptions were respected and goodness of fit was visually assessed. Then, for each color morph, another GLMM was fitted to the data to assess for morph distribution differences between top and bottom. Model outputs were analyzed using the Anova function of the package 'car' with a $\mathrm{Chi}^{2}$ statistic and Tukey Contrasts (Fox and Weisberg, 2011). All analyses were carried out in R (R Core Team, 2019). 


\section{CO1 Barcoding}

Full protocol used to confirm that both color morphs were EGA can be found as a supplementary material file to this study.

\section{Microbial community profiling}

Three adult apterous aphids of each color morph were taken from the same wheat plant from the AAFC Saskatoon Research Farm (to maximize the likelihood of obtaining differences between morphs by minimizing potential environmental or clonal variations) and total genomic DNA was extracted using a modified CTAB extraction protocol as described previously (Pérez-López et al., 2016). The genomic DNA was used as template for cpn60 universal target amplification with each reaction containing $1 \mathrm{X}$ PCR buffer (Invitrogen, Carlsbad, CA, USA), $2.5 \mathrm{mM} \mathrm{MgCl2,} 0.2 \mathrm{mM}$ each dNTP, $100 \mathrm{nM}$ each of $\mathrm{H} 279 / \mathrm{H} 280,300 \mathrm{nM}$ each of $\mathrm{H} 1612 / \mathrm{H} 1613$ and $1 \mathrm{U}$ of Platinum Taq (Invitrogen, Carlsbad, CA, USA) and cycling conditions of $1 \times 95^{\circ} \mathrm{C}, 5 \mathrm{~min} ; 40 \times 95^{\circ} \mathrm{C}, 30 \mathrm{~s}, 42-60^{\circ} \mathrm{C}, 30 \mathrm{~s}, 72^{\circ} \mathrm{C}, 30 \mathrm{~s} ; 1 \mathrm{x}, 72^{\circ}, 2 \mathrm{~min}$. Primer sequences are listed in Supplementary Table 1. Amplicons were pooled and gel purified using Blue Pippin gel electrophoresis (Sage Biosciences, Beverly, MA, USA). Purified amplicon was prepared for sequencing using the NEBNext Universal Illumina Library Prep Kit (New England Biolabs, Ipswich, MA, USA), and sequenced asymmetrically (400 forward cycles, 100 reverse cycles) using MiSeq 500 cycle v2 chemistry (Illumina, San Diego, California, United States). Sequences were assembled into operational taxonomic units using the mPUMA pipeline (Links et al., 2013) with the relative abundance of each OTU (Operational Taxonomic Unit) determined by mapping with bowtie2 v.2.3.3.1 (Langmead and Salzberg, 2012). Only full length (549bp - 561bp) assembled cpn60 OTU were retained and all sequencing libraries were rarefied to the smallest library size of 1,223,121 reads prior to analysis. BrayCurtis dissimilarity, Simpson evenness (1-D), and Shannon diversity $\left(H^{\prime}\right)$ metrics were calculated for each sample using the rarefied sequencing data. Bray-Curtis results between color morphs were tested for significance using PERMANOVA after confirming homogeneity of multivariate dispersion between groups using PERMDISP ( $p>0.05$ ). Simpson and Shannon differences between color morphs were tested using the Kruskal-Wallis test. All alpha- and beta-diversity metrics and corresponding statistical tests were calculated using the vegan package in R (Oksanen et al., 2015).

\section{Light-intensity experiments}

An EGA colony was created from the mixed-color morph 2015 field population, starting with approximately 50 aphids of each color morph. The aphid colony was raised on a mix of barley and wheat plants kept in a BugDorm 1 (Megaview Science, Taiwan) insect rearing cage, at $20 \pm 1^{\circ} \mathrm{C}, 16: 8 \mathrm{~h}$ LD (Light:Dark) photo regime, and $55 \pm 10 \%$ RH (Relative Humidity). Barley and wheat for the colony was grown in a growth chamber in 15-cm-diameter plastic pots containing soil-less mix (modified after Stringham, 1971). Approximately 250 seeds were sown per pot which yields a pot full of many green, vegetative shoots for aphid development.

Two experiments to evaluate the effect of light intensity on aphid body-color transitions were carried out in two reach-in growth cabinets (Conviron) under two different light intensities: $13 \mu \mathrm{mol} . \mathrm{m}^{-2} . \mathrm{s}^{-1}$ (low light intensity, T8 bulbs, with half a light bank illuminated) and $202 \mu \mathrm{mol} . \mathrm{m}^{-2} \cdot \mathrm{s}^{-1}$ (higher light intensity, T5 bulbs, full light bank illuminated). Both bulbs have the same color rendering properties (CRI) of 85. To provide a reference value, full sun exposure in a sunny day is $2000 \mu \mathrm{mol} . \mathrm{m}^{-2} \cdot \mathrm{s}^{-1}$ and 100 to $200 \mu \mathrm{mol} . \mathrm{m}^{-2} . \mathrm{s}^{-1}$ would be values monitored in greenhouses with artificial lights or at the bottom of 
a crop canopy. Aphids are thus likely to experience a much wider light intensity range in their natural habitat, but the tested values are standard in laboratory conditions, and were sufficient to trigger plastic responses. The temperature of $20 \pm 1^{\circ} \mathrm{C}, 16: 8 \mathrm{~h} \mathrm{LD}$ photo regime and $55 \pm 10 \% \mathrm{RH}$ were constant and similar between chambers. For the color morph experiments, an initial three wheat (c.v Roblin) seeds were planted in a 15-cm-diameter pot and the healthiest seedling in each pot was allowed to grow while the others were removed. Experiments were started when the wheat headed and aphids were added to ears after the anthesis stage and prior to the milk stage.

Green to red experiment: This experiment occurred two months after the colony was started from field-collected individuals and the colony had been kept in the low-light chamber. Groups of ten newly moulted, apterous-adult aphids (green morph EGA) were randomly chosen from the colony and were placed on ten wheat ears (10 aphids/head, $\mathrm{N}=100$ ) with crispy-wrap bags tied around the stem to confine aphids to wheat ears, and put in a new growth chamber under high-light condition. Counts of each color morph were performed after four and seven days by counting the number of green and red EGA adults and offspring. Counts continued until the majority of aphids had color-changed. Percentages of each color morph were calculated at each sample date by dividing the number of aphids of each color morph by the total number of aphids per ear.

Red to green experiment: We first needed to obtain red-morph aphids from our colony that only contained green-morphs, because it had been maintained two months under low-light conditions. To do so, one newly moulted, apterous-adult aphid (green morph EGA) was randomly chosen from the colony and placed individually onto a wheat ear $(\mathrm{N}=10)$. The individual green morph $E G A$ and their offspring rapidly shifted to predominantly $(97.9 \pm 0.8 \%$ mean proportion $\pm \mathrm{sd}$ ) red-morph color within seven days when moved from low-light to higher light conditions.

Red-morph EGA were selected at the conclusion of this short process. Then, adult red morph aphids were placed on ten wheat ears $(10 /$ head, $N=100)$ with crispy-wrap bags tied around each ear to confine aphids, and put in a new growth chamber under low-light condition. Starting four days later, aphid counts were performed every one to four days when possible up to 63 days until the majority of the aphid population had color-changed. Percentages of each color morph were calculated at each sample date by dividing the number of aphids of each color morph by the total number of aphids per ear.

Color-morph experimental data was analysed using GLMMs with the binomial error family in R, separately for the green-to-red and the red-to-green experiments. We analysed the effect of time (days) on the combined response variable of the number of green and red aphid morphs, and we used the time (days) nested in the population identity as a random effect in the models to account for the temporal dynamic in each population (i.e., in each cage). Distribution assumptions were respected and goodness of fit was visually assessed. For the green to red experiment, we used a 2nd degree polynomial for the "day", and for the red to green experiment, we used a 3rd degree polynomial (see the full model selection, Table S1). Model selection was operated using the dredge function from the MuMIn package, based on best-fit AIC (Barton and Barton, 2019). Model outputs were analyzed using the Anova function of the package 'car'. 


\section{Results}

Field study

Overall, we found less red morph than green morph aphids in the fields ( $N=3513$ green $E G A$, and $\mathrm{N}=1859$ red $E G A)$. There was an interaction effect of location with the type of morph on morph distribution on the wheat head ( $G L M M, c h i^{2}=871.2, d f=1, p<0.001$ ) (Figure 2). Red morphs EGA were mostly located at the top of the wheat ear (GLMM, chi ${ }^{2}=769.4, d f=1, p<0.001 ; 3.96 \pm 0.25$ and $6.25 \pm$ 0.32 mean \pm se aphids for bottom and top, respectively) whereas green morphs were mostly located at the bottom of the ear (GLMM, $\mathrm{chi}^{2}=181.1, \mathrm{df}=1, \mathrm{p}<0.001 ; 8.63 \pm 0.40$ and $3.24 \pm 0.24$ mean \pm se aphids for bottom and top, respectively).

\section{CO1 Barcoding}

All six aphids, three from each color morph, had CO1 barcoding regions that were $100 \%$ similar to the S. avenae $\mathrm{CO} 1$ sequences published on the Barcode of Life Data (BOLD) Database System confirming that the red and green aphids were color morphs of the EGA (S. avenae) and not distinct species of aphid.

\section{Microbial community comparison}

The microbial communities of the two aphid color morphs consisted of 136 OTU, of which 6 were likely Fungi based on BLAST comparison to the reference database cpnDB (www.cpnDB.ca) (Supplementary Table S2 and Table S3). The sequencing libraries from all insects were dominated by OTU most closely related to Buchnera sp. (59-98\% of sequencing reads) and Sphingomonas sp. (1-40\% of sequencing reads) (Figure 3A, Supplementary Table S3). The core microbiome present in the sequencing libraries for all insects was represented by bacteria from a range of phyla including Bacteroidetes, Proteobacteria, Synergistetes, Actinobacteria and Firmicutes and one fungus most similar to Cryptococcus sp. (77\% identity).

There were no significant differences between red and green aphids with regards to community diversity or evenness (Kruskal-Wallis $\mathrm{chi}^{2}=1.19, \mathrm{p}=0.28$, for both metrics) with low Shannon and Simpson 1-D values reflecting the unbalanced composition of the communities which were dominated by 2 OTUs (Figure 3B and 3C). Similarly, beta-diversity analysis based on the Bray-Curtis dissimilarity metric revealed no significant differences in the composition of the microbial communities between the two color morphs (PERMANOVA, $d f=1$, pseudo-F $=1.18 p=0.40$ ) (Figure 3D)

\section{Light-intensity experiments}

The proportion of green and red aphids significantly changed over time and aphids predominantly turned red within 72 hours when moved from low light to high light conditions. This effect was observed in both the initial population of one green-morph adult per head $(97.9 \pm 0.8 \%$ red at day 7$)$ (day effect, $\left.\mathrm{GLM}, \mathrm{chi}^{2}=231.8, \mathrm{df}=1, \mathrm{p}<0.001\right)$ and the population of ten green morph EGA $(80.8 \% \pm$ $4.5 \%$ red by day 4 ) (GLMM, day effect, $\mathrm{df}=1, \mathrm{p}<0.001, \mathrm{chi}^{2}=254.9$ and $\mathrm{chi}^{2}=27.7$, for first and second degree polynomials, respectively) (Figure 4). Offspring were included in population counts and the offspring of the initial adults also displayed the red-morph color. Some of the green morph EGA exhibited an intermediate yellow color before becoming red. According to model estimates, the shift from green to red in $50 \%$ of the aphids occurred around day 2 . 
The proportion of red and green aphids significantly changed over time (GLMM, day effect, $d f=1$, $\mathrm{p}<0.001, \mathrm{chi}^{2}=1060.4, \mathrm{chi}^{2}=885.4$ and $\mathrm{chi}^{2}=681.5$, for first, second, and third degree polynomials, respectively). When populations of red EGA were moved from high light to low light conditions, the aphid population took weeks to fully revert from red to green morph color (Figure 5). Some of the offspring of the red morph EGA exhibited an intermediate yellow color before becoming green. On average, there was a decrease of 0.62 red aphids per day and a corresponding increase of 0.62 green aphids per day over the course of the experiment. On average, $50 \%$ of the aphids had definitively turned from red to green by day 14 .

\section{Discussion}

The sampling year (summer of 2015) marked the first year that the rarer red-morph EGA was observed (or at least, reported) in cereal crops on the Canadian prairies in recent memory (Lamb and MacKay, pers. comm.). The following growing season (2016) again had both green and red morph in the same fields (Wist, pers. obs.). We found a distribution pattern of EGA on wheat ears when we observed them in the fields in Saskatchewan, Canada. Red morphs were predominantly found at the top of the wheat head, while green morphs were mostly found at the bottom. This distribution however, does not imply that red morphs were always more abundant than green morphs at the top, but only that within each color morph, the partitioning of the color-morphs on the wheat ears was distinct. Color may thus be modified by the location of the aphid on the wheat plant and may depend on the light the aphid receives and/or on the color of the plant.

The significance of such color polyphenism in EGA can first be discussed within the context of behavioral ecology and adaptive physiology. When running behavioral experiments and placing aphids onto a new plant (not shown here), we observed that most aphids walked-back to their respective location in the plant (top and bottom) within a few hours. However, the pattern was less clear than what we observed in the field. Aphids may thus choose the location on the plant that best matches their body color. Body color in the EGA has also been reported to shift along with photoperiodic changes across the year (Jenkins et al., 1999; Markkula and Rautapää, 1967). With regards to these studies and our own results, we speculate such changes may serve to synchronize the aphid coloration to changes in plant color within seasons or to the presence of predator/parasites. Color polyphenism could be camouflage behavior to hide from parasitoids and predators since the bottom of the wheat head stays greener than the top, which turns dry and yellow first. Crypsis through background matching and/or body-color change is quite common in animals and acts as anti-predator defenses (Green et al., 2019; Merilaita and Stevens, 2011; Schaefer and Stobbe, 2006; Smithers et al., 2018). For example, some octopus species have mastered the art of background mimicry to escape predation (Josef et al., 2012), although using very different mechanisms from what we observe in aphids. In chameleon prawns, crypsis is maintained by adaptively changing color slowly over days or weeks to match seasonal changes in algal cover (Green et al., 2019). Crypsis behaviors that are not related to body-color are also found in insects, such as in the aphid Eriosoma lanigerum which covers itself with wax to escape spiders (Moss et al., 2006). Switching to a reddish color could also help S. avenae aphids better resist UV exposure at the top of the plants. In all organisms, melanin and other dark pigments can limit damage incurred by exposure to UV radiations, in a way that color-change by production of such pigments is highly plastic (Majerus, 1998; True, 2003). In aphids, Hu et al. (2013) demonstrated 
that the red-morph EGA exhibited stronger adaptability (e.g., better growth rate, better longevity) than the green morph at high doses of UV-B exposure. More studies are needed to confirm if this color polyphenism is an adaptive response in the field, in which case we could suspect selection on plasticity, or simply the product of environmental constraints. For example, coloration and background matching would have to be precisely quantified. It could also be interesting to perform behavioral experiments to see if the coloration and the cryptic behavior reduces parasitism or predation rates on the EGA, as it does in the pea aphid system (Dion et al., 2011; Polin et al., 2015), and to account for the great diversity of predator visual systems to test the adaptive hypothesis further (Théry and Gomez, 2010).

We provided some evidence that the bacterial community within S. avenae aphids was very simple and dominated by only two OTUs; most closely related to the obligatory symbiont Buchnera $s p$. (89\% nucleotide identity) (Oliver et al., 2014) and Sphingomonas sp. (89\% nucleotide identity) commonly associated with phloem-feeding insects (Gallo-Franco et al., 2019). Some of the detected microorganisms, including Cryptococcus, have been found previously to be associated with several species of aphid (Grigorescu et al., 2018). This simple community dominated by very few OTU was reflected in very low diversity metrics with no significant differences between communities from the two color morphs. Additionally, the Bray-Curtis dissimilarity metric showed no difference in microbial community composition between red and green morph EGA in terms of the number and relative abundance of shared microorganisms. As also pointed out by Alkhedir et al. (2010), no genes in the fully sequenced genome of the obligatory symbiont Buchnera have been identified coding for carotenoids, nor in two facultative symbionts (Moran and Jarvik, 2010). However, one can stress that it is not necessarily the most abundant bacteria that causes the coloration effect. We found no OTU closely related to Rickettsiella sp. symbionts in any of the two morphs. Yet, this endosymbiont is known to be responsible for body coloration of the pea aphid A. pisum (Tsuchida et al., 2010). Therefore, and despite the few aphid individuals that we could sequence with the Illumina platform, we argue that body color in $S$. avenae is likely not due to different endosymbiotic bacteria that make a colored pigment. However, we cannot exclude that it is due to differential levels of pigment production by a given strain under different environmental conditions, or even by genotypic interactions with the endosymbiont depending on the aphid clone. Using aposymbiotic lineages of aphids under different conditions of light-intensities would help exploring the question of morphs coloration more in depth.

We showed that light-intensity is a good candidate for explaining differential color in EGA; both red and green morph EGA could be obtained at proportions close to $90-95 \%$ when exposed to either high or low intensity lights, respectively. The observed polyphenism in the field and laboratory is most likely due to a plastic process in response to light, that makes aphid body color shift along a spectrum from green to red, and does not seem to be linked to any kind of genetic polymorphism (as it is suspected in the pea aphid (Caillaud and Losey, 2010)). We thus suggest that, depending on their location on the plant, aphids receive different light intensities so they become differentially colored. As we tested for light-intensities that were lower than what aphids are likely to encounter in the field, probably the shift from low to higher values of light intensity (or reversely) is more important to trigger plasticity than the absolute value of light intensity perceived. Our findings are consistent with the results of Alkhedir et al. (2010), who showed that red color morph formation was controlled by light. How exposure to high-light intensity triggers the pigment-production cascade in insects remains to be studied. We additionally show that color can be rapidly (i.e., within the lifespan of an individual aphid) reversed from a color to another, although the switch from green to red (around 7 days) was much faster than from red to green (around 60 days). It is very likely that being able to rapidly switch from 
green to red is adaptive under conditions of prolonged periods of sunlight during summer, for example. One could speculate that aphids' "baseline" color is green and they would produce a significant quantity of carotenoids to quickly become red when exposed to high light-intensities. Carotenoid production is however likely to cost energy and other resources, as it is the case for most pigment production in animals (Roff and Fairbairn, 2013; Stoehr, 2006), so any advantage would have to balance the costs. Conversely, it might be physiologically constraining and take more time to eliminate carotenoids from the hemolymph and tegument, to reverse the color back from red to green (Kayser, 1982; Shamim et al., 2014). It could also simply not be time-constrained, because if costly pigment production stops, then both color-morphs would hypothetically be as performant under low-light conditions. In summer, when we sampled the aphids, light intensity is higher than when closer to fall, or spring, which would favor the production of red-morph EGA, according to our light experiment results. This is in line with the observations of Chroston (1983) who mentioned that green morphs prevailed in fall or spring, and red-morphs in summer. However, we observed twice as many green aphids than red aphids on the wheat ears we sampled during the summer of 2015. Green morphs are known to produce more alates and disperse more than red morph EGAs (Ankersmit and Dijkman, 1983), which could explain the prevalence of green morphs in the short summer window of the growing season in Saskatchewan.

Although we were not able to track color changes at the individual scale, our results suggest that rapid and multiple color shifts could have occurred. For example, on Figure 5 (red-to-green shift), we observed that for some populations, most aphids turned green around day 7 , then turned back to red at day 8 before turning green again progressively until the end of the experiment. This pattern could be due to either very rapid plastic response, to stochastic changes in color, or to relative change in aphid numbers in each population due to offspring production of different colors and mortality. Alkhedir et al. (2010) observed clonal differences in the capacity to change color in response to light intensity in the EGA. Our study was focused at the population level, so we do not have information for each clone. However, in proportion, most aphids changed color, as well as their offspring, when exposed to a different light intensity. This pattern may highlight poor clonal diversity in aphids from northern limits of cultivable areas, such as Saskatchewan, where populations are likely to show a pioneering effect from Northward migration each year due to harsh winters where EGA do not survive (Van Baaren et al., 2019).

To conclude, S. avenae is an additional example among the animal species whose body color has been observed to change and to be reversible within individuals in response to environmental conditions. Particularly, we showed the importance of light-intensity for triggering the plastic response of colorchange over the lifespan of an aphid. Our study, among many others, helps highlighting the diversity of mechanisms underlying animal body-color variations, from genetic polymorphism within populations to fast plastic changes at the individual scale. Such results also show that body-color is not necessarily predetermined during early phases of ontogeny and can, in some instances, shift during the entire lifespan of an individual. Studies considering the adaptive significance of such fast plastic color changes within individuals in the field will help better understanding their evolutionary and ecological importance in animals. 


\section{Acknowledgments}

The authors thank Agriculture and Agri-Food Canada for offering vehicles for field data collection and growth cabinet space to rear the aphids. We acknowledge permission from landowners of the commercial fields where research was done. We thank the three anonymous reviewers who provided valuable and constructive feedback on an earlier version of this manuscript.

\section{Funding}

This research was funded by the FP7-PEOPLE-2013-IRSES program (project APHIWEB, grant no. 611810 to Nicolas Desneux (coordinator) and Joan van Baaren and Kévin Tougeron (participants)) and through AAFC A-base funding provided to Tyler Wist through the Pest Management Centre for project PRR15040. Kévin Tougeron was supported by the Fyssen foundation and the F.R.S.-FNRS during the writing process of the article. This article is number BRC 368 of the Biodiversity Research Center of the UCLouvain.

\section{Conflict of interest}

The authors declare that they have no conflict of interest.

\section{Figures}

Figure 1: A. Picture of a wheat head showing both green and red morph Sitobion avenae. Black arrows indicate start and end position of aphid counts in the field experiment. B. Green and red female morphs of Sitobion avenae sampled in wheat fields in Saskatchewan, Canada (photo credits Kevin Tougeron CC-BY-NC). 
A.

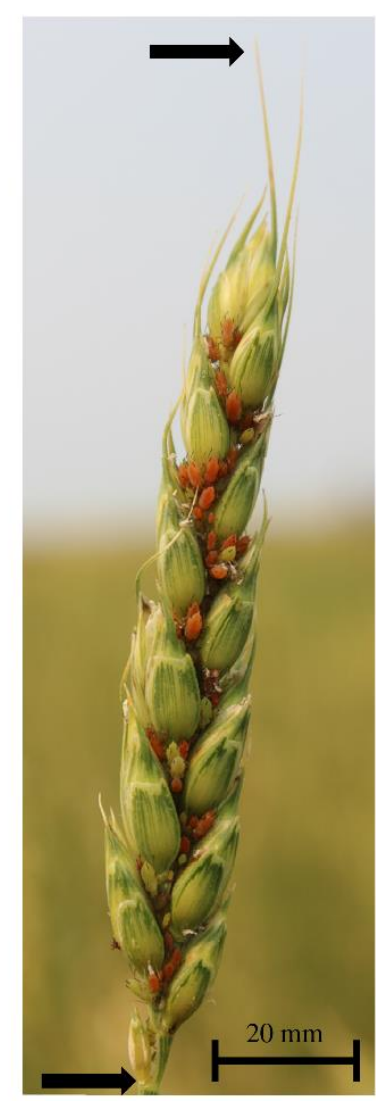

B.

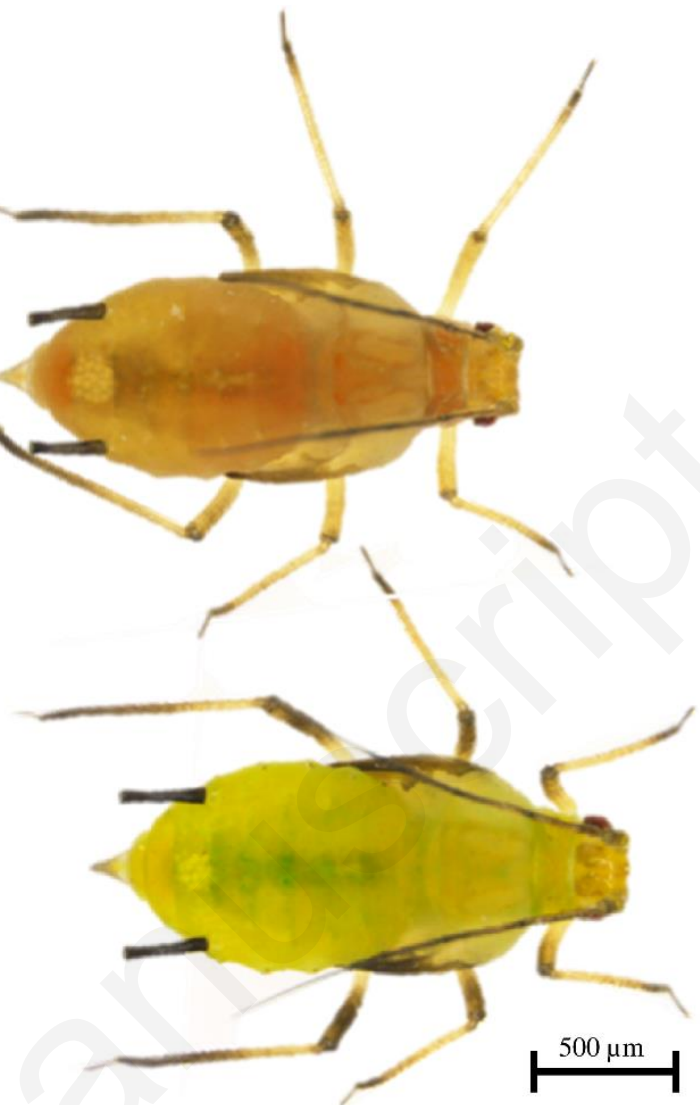

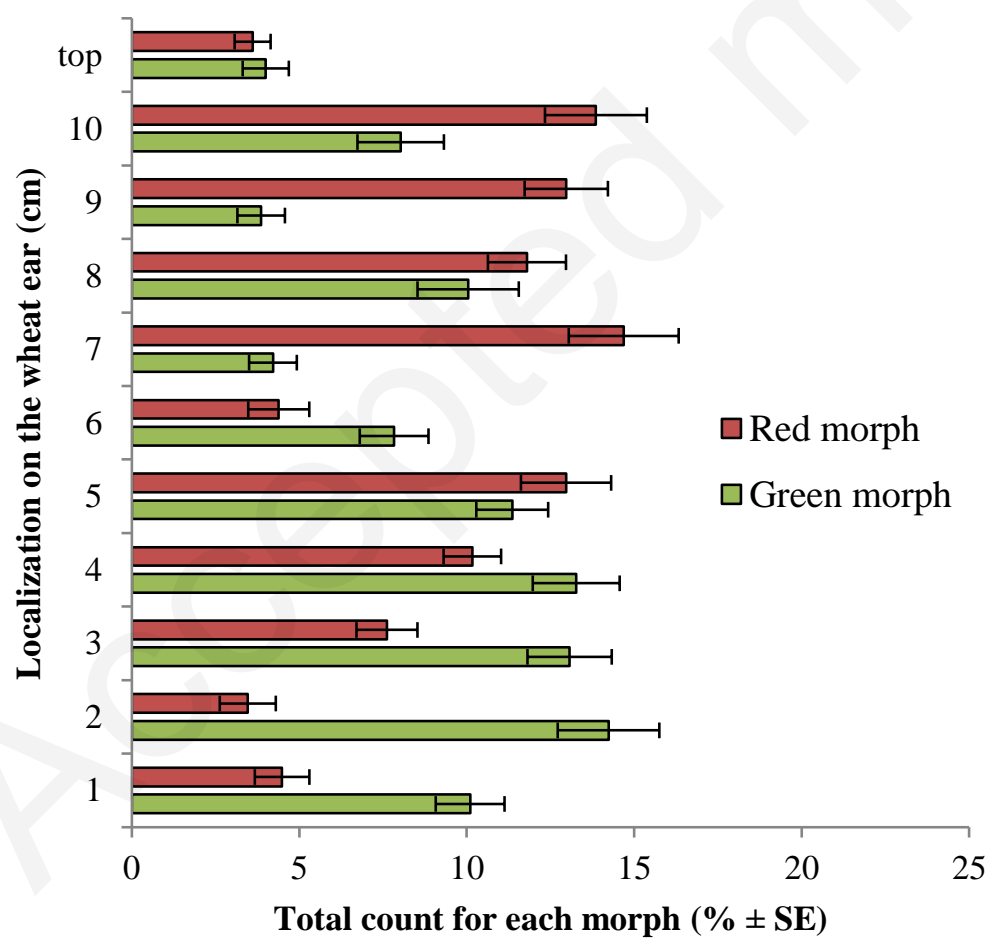

Figure 2: Proportion of each Sitobion avenae morph (green and red bars) at different locations of the wheat ear (from $1 \mathrm{~cm}$ from the base to the top). Total of 65 sampled wheat heads, $\mathrm{N}=3513$ green EGA and $\mathrm{N}=1859$ red $\mathrm{EGA}$. 
A

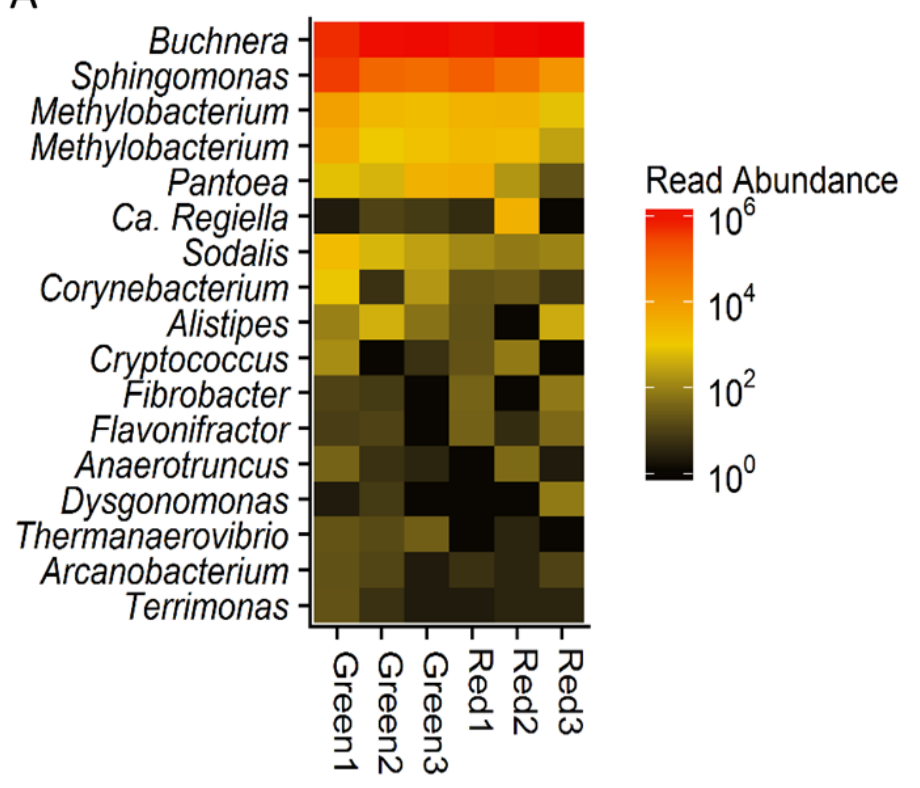

B

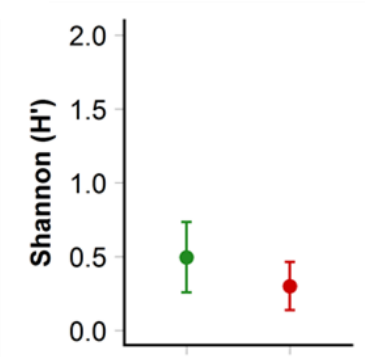

D

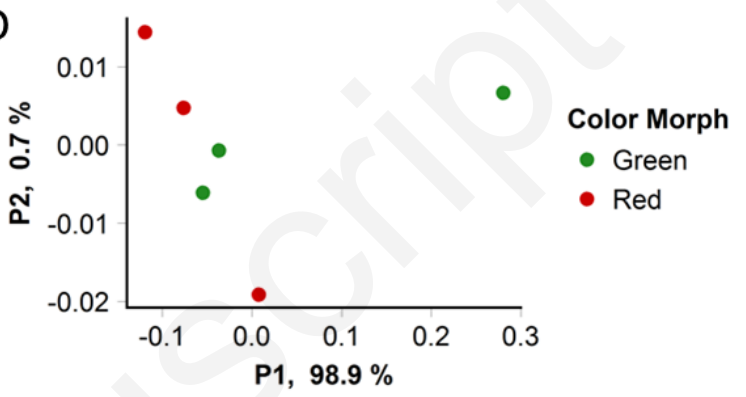

Figure 3: (A) Heatmap of abundances of the core microbiome for three green and three red morphs of Sitobion avenae. Each row represents an assembled operational taxonomic unit (OTU) labelled according to the nearest neighbor genus based on BLAST alignment to the reference database cpnDB. On the right panel, rarefied OTU frequency data $(1,223,121$ reads per sample) was used as input for calculating (B) Shannon diversity, (C) Simpson evenness and (D) Bray-Curtis dissimilarity metrics for microbial community profiles generated from aphid morphs with red or green body-color.

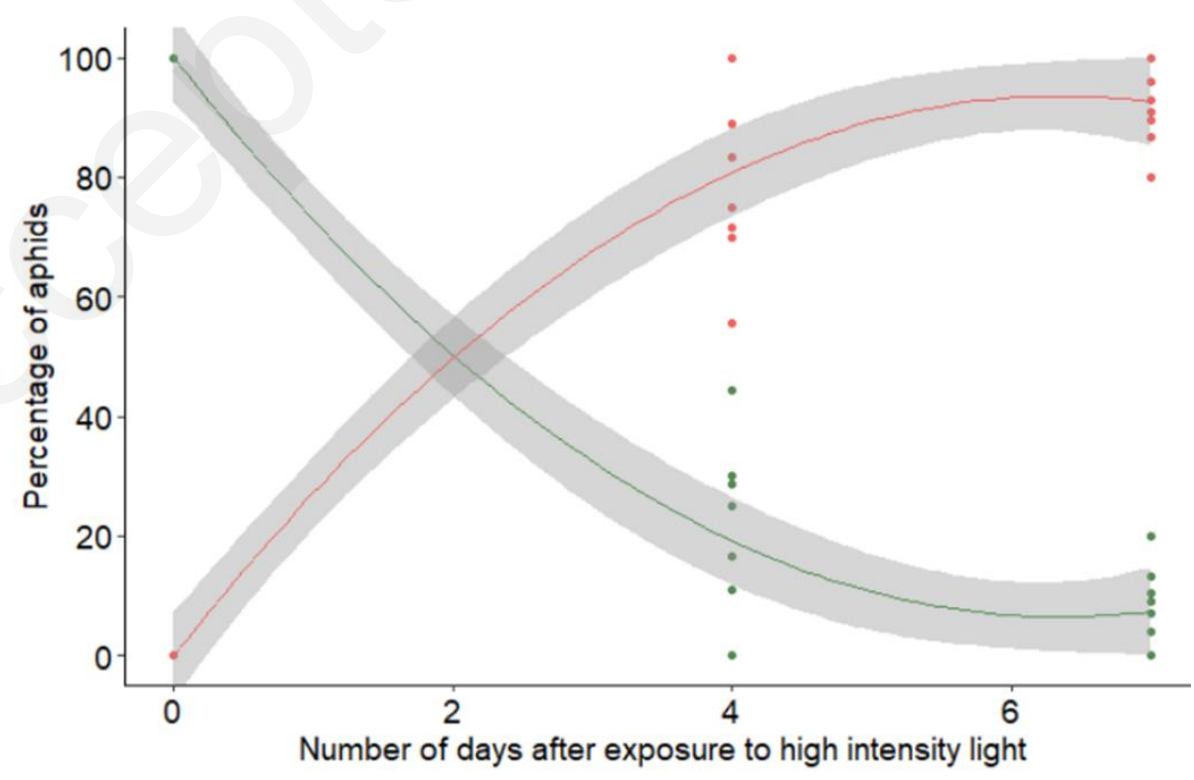


Figure 4: Color morph shift from green (green dots) to red (red dots) of a population of English grain aphids moved from low to high light conditions, monitored over 7 days. As represented on this figure, this transition results in a mirror image shift among morphs as each dot represents an aphid population monitored over time. $\mathrm{N}=10$ populations. Note that some data points may be overlapping. Smooth envelopes represent $95 \% \mathrm{Cls}$ around the non-linear regression line $\left(2^{\text {nd }}\right.$ degree polynomial, best AIC fit).

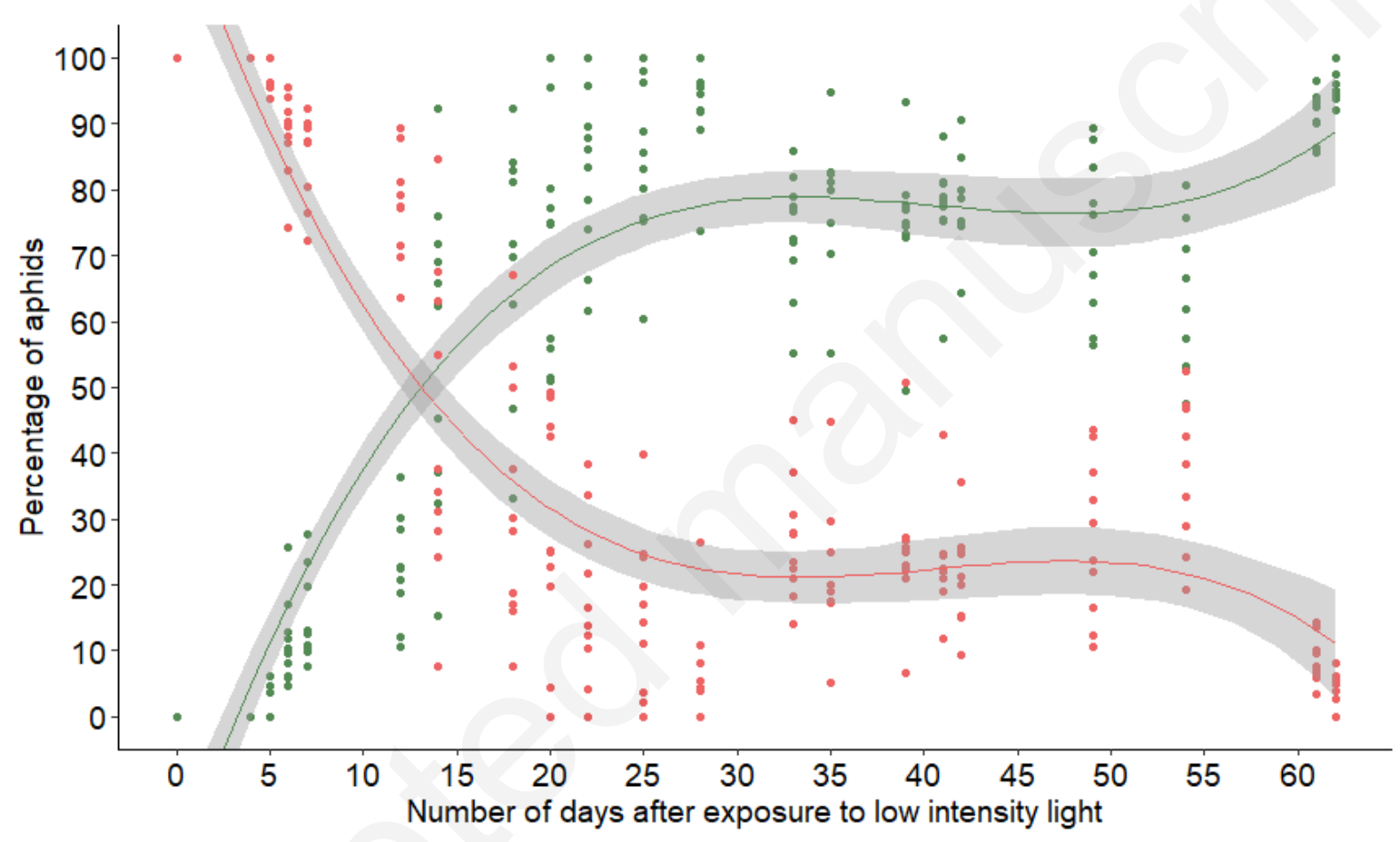

Figure 5: Color morph shift from red (red dots) to green (green dots) of a population of English grain aphids moved from high to low light conditions, monitored over 63 days. As represented on this figure, this transition results in a mirror image shift among morphs as each dot represents an aphid population monitored over time. $\mathrm{N}=10$ populations. Note that some data points may be overlapping. Smooth envelopes represent $95 \% \mathrm{Cls}$ around the non-linear regression line ( $3^{\text {rd }}$ degree polynomial, best AIC fit). 


\section{Supplementary Material}

\section{CO1 barcoding method}

Three individual EGA of each color morph were taken from six different wheat heads in the same field (AAFC Melfort Research Farm) and "barcoded" by sequencing a 658-bp region of their mitochondrial CO1 gene, to confirm that both color morphs were EGA (Hebert et al., 2003). Sequences were compared against those published on the Barcode of Life Data (BOLD) Database. DNA was extracted from three aphids of each color taken using a QIAGEN ${ }^{\circ}$ DNeasy ${ }^{\circ}$ Blood and Tissue Kit. Using IDT ${ }^{\text {tm }}$ primers LCO1490 and HCO2198, a 658-bp region of the CO1 gene was amplified with a $25 \mu \mathrm{L}$ PCR using reagents sourced from Invitrogen ${ }^{\mathrm{m} m}$. PCR reaction conditions were $94^{\circ} \mathrm{C}$ for 1 minute as an initial denaturing step, followed by five cycles at $94^{\circ} \mathrm{C}$ for 40 seconds, $45^{\circ} \mathrm{C}$ for 40 seconds, and $72^{\circ} \mathrm{C}$ for 1 minute for denaturation, another 35 cycles at $94^{\circ} \mathrm{C}$ for 40 seconds, $51^{\circ} \mathrm{C}$ for 40 seconds, and $72^{\circ} \mathrm{C}$ for 1 minute for annealing, then $72^{\circ} \mathrm{C}$ for 5 minutes for extension, and finally held at $4^{\circ} \mathrm{C}$ indefinitely. The PCR product was run on a $1.5 \%$ agarose gel to confirm the amplification of the CO1 gene. The PCR product was purified using QIAGEN $^{\circ}$ QIAquick $^{\circ}$ PCR Purification Kit and DNA concentrations were measured using an Invitrogen ${ }^{\text {tm }}$ Qubit $^{\text {tm }}$ dsDNA HS assay kit and a Qubit ${ }^{\text {tm }} 2.0$ fluorometer, and then sequenced by Eurofins Genomics. Sequencing results were cleaned and aligned using Vector NTI Advance 11.5.4. Generated molecule sequences were compared against those published on the Barcode of Life Database (BOLD) and BLAST databases for results comparison and solidarity. Results with both the highest percent match probability and specificity were recorded. 
Table S1: Model selection table ("dredge" function from the MuMin package) for the green-to-red and the red-to-green color change experiments. Model input is the number of aphid and explanatory variables are the number of days after the beginning of the experiment, using a 3rd degree polynomial for the green-to-red experiment, and using a quadratic polynomial for the red-to-green experiment (data with 3 monitored days did not allow testing a 3rd degree polynomial function). Selected models are in bold, for each experiment.

\begin{tabular}{|c|c|c|c|c|c|c|c|c|c|c|c|c|c|c|c|}
\hline \multirow[b]{2}{*}{ Model } & \multicolumn{8}{|c|}{ Green-to-red } & \multicolumn{7}{|c|}{ Red-to-green } \\
\hline & Intercept & $x$ & $x^{2}$ & $x^{3}$ & $d f$ & Log Lik. & $\mathrm{AlCc}$ & Delta AIC & Intercept & $x$ & $x^{2}$ & $\mathrm{df}$ & Log Lik. & $\mathrm{AlCc}$ & Delta AIC \\
\hline$\sim x+x^{2}+x^{3}$ & 22.8 & -0.8 & 391.2 & -204.5 & 7 & -970.9 & 1956.5 & 0 & / & I & / & & / & / & / \\
\hline$\sim x+x^{2}$ & 7.2 & -0.3 & 58.9 & / & 6 & -1400.9 & 2814.4 & 857.9 & -37.5 & 8.7 & -84.4 & 3 & -29.9 & 66.8 & 0 \\
\hline$\sim x$ & 1.0 & -0.04 & / & / & 5 & -2088.7 & 3166.5 & 2231.2 & -2.8 & 0.9 & / & 2 & -43.8 & 92.0 & 25.2 \\
\hline null & -0.2 & / & / & / & 4 & -2100.9 & 4210.2 & 2253.7 & 0.7 & / & / & 1 & -183.9 & 369.9 & 303.2 \\
\hline
\end{tabular}

Table S2: Primer sequences used for microbial community profiling

\begin{tabular}{ll}
\hline Primer Name & Primer Sequence (5'-3') \\
\hline H279 & GAIIIIGCIGGIGAYGGIACIACIAC \\
H280 & YKIYKITCICCRAAICCIGGIGCYTT \\
H1612 & GAIIIIGCIGGYGACGGYACSACSAC \\
H1613 & CGRCGRTCRCCGAAGCCSGGIGCCTT \\
\hline
\end{tabular}


Table S3: Complete OTU frequencies with BLAST results on three green morphs and three red morphs EGA. The core microbiome (OTU observed in all samples) is highlighted in bold.

\begin{tabular}{|c|c|c|c|c|c|c|c|c|c|}
\hline \#OTU & Green1 & Green2 & Green3 & Red1 & Red2 & Red3 & Length & \%BlastID & BLAST Match \\
\hline c1129_g1_i1 & 717377 & 1099097 & 1122330 & 1046260 & 1147629 & 1196735 & 579 & 91.06 & AF003957 Buchnera sp. \\
\hline c1129_g2_i1 & 486635 & 112266 & 90152 & 163183 & 62677 & 14871 & 579 & 94.39 & NZ_ATTI01000001 Sphingomonas phyllosphaerae \\
\hline c1964_g1_i1 & 8361 & 2424 & 1933 & 3116 & 3378 & 777 & 579 & 81.42 & NC_007406 Nitrobacter winogradskyi \\
\hline c842_g1_i1 & 4905 & 1042 & 1545 & 2359 & 2125 & 290 & 591 & 81.8 & NZ_ALIQ01000058 Chelatococcus sp. \\
\hline c1411_g1_i1 & 738 & 512 & 3430 & 4385 & 181 & 17 & 590 & 100 & NZ_ADWZ01000005 Pantoea sp. \\
\hline c350_g1_i1 & 2 & 9 & 7 & 4 & 3484 & 1 & 587 & 94.41 & GL379589 Candidatus Regiella \\
\hline c1984_g7_i1 & 2128 & 550 & 269 & 124 & 81 & 94 & 591 & 90.74 & AF326971 Sodalis glossinidius \\
\hline c1964_g2_i2 & 9 & 1 & 142 & 990 & 57 & 1 & 617 & 86.69 & NSIL01000016 Acidobacterium sp. \\
\hline c1873_g2_i2 & 920 & 8 & 195 & 18 & 24 & 5 & 619 & 96.47 & NZ_ACVP01000037 Corynebacterium tuberculostearicum \\
\hline c1711_g1_i1 & 90 & 456 & 57 & 17 & 1 & 395 & 592 & 85.79 & NZ_KB905695 Proteiniphilum acetatigenes \\
\hline c2005_g1_i1 & 136 & 1 & 5 & 17 & 82 & 1 & 632 & 84.99 & AAEY01000007 Cryptococcus neoformans \\
\hline c1900_g1_i1 & 11 & 5 & 1 & 34 & 1 & 68 & 581 & 80.15 & AY017380 Fibrobacter succinogenes \\
\hline c67_g1_i1 & 6 & 10 & 1 & 31 & 6 & 39 & 583 & 85.09 & NZ_KB899665 Paenibacillus daejeonensis \\
\hline c1978_g10_i1 & 34 & 6 & 3 & 1 & 46 & 2 & 575 & 87.07 & AAXG02000005 Pseudoflavonifractor capillosus \\
\hline c1863_g3_i1 & 2 & 7 & 1 & 1 & 1 & 70 & 604 & 84.07 & AP018040 Petrimonas sp. \\
\hline c144_g1_i1 & 19 & 10 & 28 & 1 & 3 & 1 & 574 & 83.52 & ACUH01000047 Synergistes sp. \\
\hline c1398_g1_i1 & 17 & 8 & 2 & 5 & 2 & 9 & 581 & 84.33 & NZ_KV793355 Trueperella sp. \\
\hline c107_g1_i1 & 17 & 5 & 2 & 1 & 2 & 4 & 598 & 89.47 & NZ_NFUV01000001 Chitinophagaceae bacterium \\
\hline c1962_g1_i2 & 13 & 1718 & 124 & 7 & 0 & 2680 & 588 & 91.14 & NZ_ATXQ01000008 Pseudomonas caeni \\
\hline c1962_g1_i1 & 40 & 960 & 71 & 0 & 9 & 689 & 588 & 89.3 & NZ_ATXQ01000008 Pseudomonas caeni \\
\hline c1783_g3_i1 & 152 & 572 & 1 & 0 & 0 & 961 & 593 & 85.06 & AB510684 Bacteroides plebeius \\
\hline c1970_g3_i1 & 311 & 1 & 0 & 1133 & 0 & 0 & 573 & 83.82 & NC_013523 Sphaerobacter thermophilus \\
\hline c1964_g5_i1 & 7 & 0 & 71 & 3 & 904 & 0 & 571 & 86.57 & NZ_LT629749 Friedmanniella luteola \\
\hline c1967_g1_i1 & 0 & 378 & 42 & 0 & 0 & 296 & 569 & 88.61 & ACEC01000026 Clostridium methylpentosum \\
\hline c1962_g2_i1 & 1 & 278 & 2 & 6 & 0 & 416 & 587 & 82.73 & NC_015500 Treponema brennaborense \\
\hline
\end{tabular}




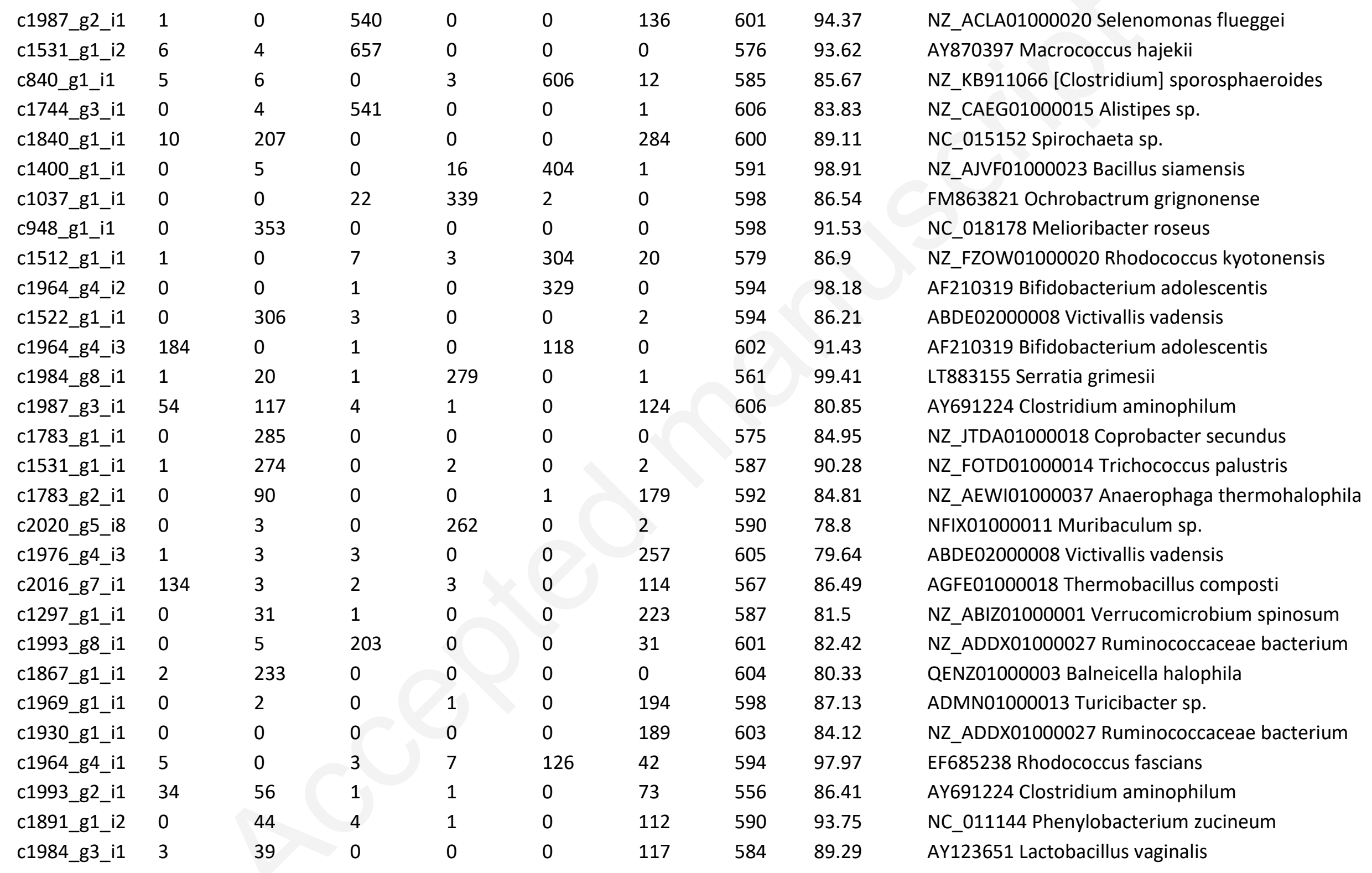




$\begin{array}{lllllllll}\text { c1724_g1_i1 } & 0 & 157 & 0 & 0 & 0 & 0 & 604 & 91.07 \\ \text { c349_g1_i1 } & 0 & 10 & 0 & 147 & 0 & 0 & 599 & 80.71 \\ \text { c2020_g5_i4 } & 2 & 12 & 0 & 0 & 0 & 137 & 608 & 81.03 \\ \text { c851_g1_i1 } & 143 & 0 & 0 & 0 & 0 & 0 & 584 & 100 \\ \text { c1991_g1_i2 } & 43 & 22 & 0 & 0 & 0 & 74 & 563 & 84.44 \\ \text { c1891_g1_i1 } & 0 & 7 & 116 & 0 & 1 & 13 & 603 & 79.92 \\ \text { c1997_g2_i1 } & 2 & 15 & 1 & 0 & 0 & 117 & 578 & 84.01 \\ \text { c1681_g1_1 } & 2 & 0 & 0 & 0 & 132 & 0 & 581 & 84.92 \\ \text { c1137_g1_i1 } & 31 & 0 & 8 & 78 & 12 & 1 & 653 & 84.5 \\ \text { c1309_g1_i1 } & 1 & 0 & 129 & 0 & 0 & 0 & 579 & 85.22 \\ \text { c1800_g2_i2 } & 68 & 0 & 10 & 11 & 37 & 1 & 574 & 90.51 \\ \text { c1800_g2_i1 } & 118 & 3 & 0 & 2 & 0 & 1 & 577 & 99.82 \\ \text { c1795_g1_1 } & 0 & 8 & 0 & 0 & 0 & 99 & 588 & 81.82 \\ \text { c1907_g1_i1 } & 1 & 0 & 4 & 0 & 2 & 97 & 570 & 92.75 \\ \text { c1472_g1_i1 } & 0 & 0 & 0 & 0 & 0 & 103 & 587 & 81.01 \\ \text { c1896_g1_i1 } & 0 & 19 & 0 & 0 & 0 & 84 & 598 & 82.95 \\ \text { c2020_g5_i2 } & 0 & 6 & 0 & 0 & 0 & 92 & 607 & 82.12 \\ \text { c876_g1_i1 } & 0 & 2 & 0 & 0 & 96 & 0 & 592 & 85.59 \\ \text { c1683_g1_i1 } & 0 & 0 & 0 & 0 & 0 & 97 & 608 & 97.87 \\ \text { c2020_g5_i6 } & 0 & 6 & 0 & 0 & 0 & 87 & 590 & 81.77 \\ \text { c1641_g1_i1 } & 0 & 2 & 6 & 0 & 0 & 84 & 601 & 88.24 \\ \text { c1981_g2_i1 } & 2 & 1 & 0 & 1 & 0 & 88 & 592 & 87.39 \\ \text { c1993_g9_i2 } & 3 & 8 & 0 & 0 & 78 & 0 & 568 & 94.5 \\ \text { c1976_g4_i2 } & 1 & 5 & 1 & 0 & 0 & 81 & 578 & 91.78 \\ \text { c1923_g1_i1 } & 62 & 1 & 0 & 0 & 0 & 21 & 598 & 84.3 \\ \text { c1989_g1_i1 } & 0 & 1 & 82 & 0 & 1 & 0 & 590 & 83.4 \\ \text { c1554_g1_i1 } & 0 & 9 & 0 & 0 & 0 & 70 & 604 & 83.75 \\ \text { c1993_g9_i1 } & 0 & 0 & 0 & 0 & 13 & 66 & 602 & 93.61 \\ \text { c1202_g1_i1 } & 3 & 70 & 2 & 1 & 0 & 0 & 578 & 78.57\end{array}$

CP002292 Rhodomicrobium vannielii

NZ_FTRU01000008 Negativibacillus massiliensis NFIX01000011 Muribaculum sp.

AF286735 Bifidobacterium lactis

NZ_FNHQ01000030 Megasphaera paucivorans NC_006177 Symbiobacterium thermophilum NZ_OUNG01000001 Solobacterium sp. AAWL01000019 Thermosinus carboxydivorans FJ973632 Trametes versicolor CP000386 Rubrobacter xylanophilus KB209926 Ophiostoma novo-ulmi KB209926 Ophiostoma novo-ulmi QKZK01000001 Cytophaga xylanolytica ACUH01000047 Synergistes sp.

NZ_HE997186 Phocaeicola abscessus NZ_KB894541 Alistipes onderdonkii NFIX01000011 Muribaculum sp. NZ_QAYX01000020 Opitutus sp. AY691209 Bacteroides caccae NFIX01000011 Muribaculum sp. AY691283 Prevotella heparinolytica AY837563 Desulfobacterium autotrophicum AECU01000014 Faecalibacterium cf. CP001114 Deinococcus deserti NZ_ADDX01000027 Ruminococcaceae bacterium ABOX02000008 Bacterium sp.

AB510684 Bacteroides plebeius AECU01000014 Faecalibacterium cf. AY691219 Clostridium papyrosolvens 


$\begin{array}{ll}\text { c941_g1_i1 } & 0 \\ \text { c1717_g1_i1 } & 0 \\ \text { c1624_g1_i1 } & 0 \\ \text { c1970_g5_i1 } & 1 \\ \text { c1479_g1_i1 } & 26 \\ \text { c1997_g1_i1 } & 0 \\ \text { c2020_g8_i2 } & 0 \\ \text { c1744_g2_i3 } & 0 \\ \text { c1978_g6_i1 } & 43 \\ \text { c1991_g2_i1 } & 0 \\ \text { c1568_g1_i1 } & 0 \\ \text { c1305_g1_i1 } & 1 \\ \text { c1599_g1_i1 } & 25 \\ \text { c1993_g7_i1 } & 0 \\ \text { c2020_g5_i5 } & 0 \\ \text { c1970_g11_i1 } & 0 \\ \text { c392_g1_i1 } & 1 \\ \text { c1456_g1_i1 } & 0 \\ \text { c2563_g1_i1 } & 13 \\ \text { c348_g1_i1 } & 0 \\ \text { c1591_g2_i1 } & 0 \\ \text { c1111_g1_i1 } & 2 \\ \text { c2020_g5_i1 } & 0 \\ \text { c1895_g1_i1 } & 0 \\ \text { c2020_g4_i1 } & 0 \\ \text { c2020_g5_i7 } & 1 \\ \text { c821_g1_i1 } & 0 \\ \text { c1347_g2_i1 } & 1 \\ \text { c1991_g1_i1 } & 5\end{array}$

\begin{tabular}{|c|c|}
\hline 0 & 0 \\
\hline 0 & 0 \\
\hline 5 & 0 \\
\hline 0 & 62 \\
\hline 0 & 0 \\
\hline 3 & 0 \\
\hline 0 & 0 \\
\hline 1 & 52 \\
\hline 1 & 8 \\
\hline 0 & 0 \\
\hline 12 & 1 \\
\hline 0 & 1 \\
\hline 29 & 1 \\
\hline 6 & 0 \\
\hline 4 & 0 \\
\hline 0 & 13 \\
\hline 0 & 0 \\
\hline 1 & 0 \\
\hline 0 & 1 \\
\hline 21 & 31 \\
\hline 32 & 0 \\
\hline 4 & 1 \\
\hline 7 & 0 \\
\hline 0 & 0 \\
\hline 0 & 0 \\
\hline 1 & 0 \\
\hline 0 & 0 \\
\hline 1 & 0 \\
\hline 2 & 0 \\
\hline
\end{tabular}

80.24

83.88

89.33

87.5

81.93

80.83

82.38

82.23

80.86

89.33

83.2

86.67

80

87.5

82.78

87.83

86.93

80.47

91.01

83.53

82.34

84.17

79.45

82.25

82.99

80.3

79.78

83.88

92.47
ACUH01000047 Synergistes sp.

ABVR01000041 Coprococcus comes

CM001403 Mucilaginibacter paludis

NZ_JOFO01000074 Microtetraspora glauca

AAEY01000007 Cryptococcus neoformans

NZ_OUNG01000001 Solobacterium sp.

NFIX01000011 Muribaculum sp.

NZ_KB894541 Alistipes onderdonkii

NZ_CP007130 Gemmatirosa kalamazoonesis

NZ_MPTA01000010 Paenisporosarcina indica

AY691256 Eubacterium ventriosum

AKWV02000084 Leptospira sp.

NC_014225 Waddlia chondrophila

NZ_ABGD02000012 Anaerotruncus colihominis NFIX01000011 Muribaculum sp.

NC_013523 Sphaerobacter thermophilus

QEKY01000009 Porphyromonas loveana

OCMV01000002 Ruminiclostridium thermocellum

NZ_QLMH01000024 Anoxybacillus vitaminiphilus

NZ_FPCJ01000001 Crenotalea thermophila

NZ_KB905695 Proteiniphilum acetatigenes

AB510681 Bacteroides massiliensis

NFIX01000011 Muribaculum sp.

NC_012489 Gemmatimonas aurantiaca

NFIX01000011 Muribaculum sp.

NFIX01000011 Muribaculum sp.

NZ_ABGD02000012 Anaerotruncus colihominis

ABDE02000008 Victivallis vadensis

NZ_FNHQ01000030 Megasphaera paucivorans 


$\begin{array}{ll}\text { c1407_g1_i1 } & 0 \\ \text { c1978_g6_i2 } & 11 \\ \text { c991_g1_i1 } & 0 \\ \text { c242_g1_i1 } & 1 \\ \text { c1777_g1_i1 } & 0 \\ \text { c560_g1_i1 } & 0 \\ \text { c1400_g2_i1 } & 34 \\ \text { c1976_g4_i1 } & 0 \\ \text { c1989_g3_i1 } & 34 \\ \text { c1989_g3_i2 } & 4 \\ \text { c1965_g3_i1 } & 0 \\ \text { c1976_g6_i1 } & 15 \\ \text { c545_g1_i1 } & 0 \\ \text { c101_g1_i1 } & 2 \\ \text { c2020_g9_i1 } & 0 \\ \text { c1157_g1_i1 } & 0 \\ \text { c1187_g1_i1 } & 0 \\ \text { c139_g1_i1 } & 0 \\ \text { c195_g1_i1 } & 6 \\ \text { c1970_g8_i1 } & 1 \\ \text { c419_g1_i1 } & 0 \\ \text { c1970_g8_i2 } & 0 \\ \text { c2020_g8_i1 } & 0 \\ \text { c1744_g2_i1 } & 0 \\ \text { c1976_g3_i1 } & 0 \\ \text { c258_g1_i1 } & 15 \\ \text { c2782_g1_i1 } & 19 \\ \text { c2020_g5_i3 } & 0 \\ \text { c2121_g1_i1 } & 0\end{array}$

$\begin{array}{ll}0 & 0 \\ 0 & 27 \\ 7 & 34 \\ 1 & 38 \\ 5 & 0 \\ 38 & 0 \\ 2 & 0 \\ 32 & 3 \\ 0 & 1 \\ 0 & 5 \\ 0 & 12 \\ 1 & 0 \\ 0 & 0 \\ 1 & 2 \\ 0 & 0 \\ 27 & 0 \\ 1 & 25 \\ 0 & 0 \\ 10 & 1 \\ 0 & 10 \\ 0 & 0 \\ 0 & 3 \\ 8 & 0 \\ 16 & 4 \\ 17 & 0 \\ 1 & 0 \\ 0 & 0 \\ 11 & 0 \\ 3 & 1 \\ & \\ 0 \\ 0\end{array}$

$\begin{array}{llll}41 & 0 & 582 & 90.38 \\ 3 & 0 & 600 & 80.25 \\ 0 & 0 & 578 & 89.89 \\ 0 & 0 & 589 & 83.76 \\ 0 & 34 & 589 & 81 \\ 0 & 0 & 581 & 83.64 \\ 0 & 1 & 583 & 94.12 \\ 0 & 2 & 567 & 85.82 \\ 2 & 0 & 566 & 84.98 \\ 26 & 0 & 591 & 87.25 \\ 0 & 0 & 572 & 88.79 \\ 2 & 0 & 588 & 91.12 \\ 0 & 32 & 590 & 84.66 \\ 0 & 24 & 594 & 84.43 \\ 0 & 30 & 575 & 84.96 \\ 0 & 0 & 576 & 94.86 \\ 0 & 1 & 596 & 86.9 \\ 0 & 27 & 586 & 85.59 \\ 0 & 2 & 570 & 84.29 \\ 13 & 0 & 597 & 87.31 \\ 0 & 23 & 567 & 82.74 \\ 18 & 0 & 595 & 87.25 \\ 0 & 13 & 593 & 79.85 \\ 0 & 0 & 597 & 81.33 \\ 0 & 2 & 548 & 86.89 \\ 2 & 2 & 566 & 82.89 \\ 0 & 0 & 600 & 96.53 \\ 0 & 7 & 583 & 83.72 \\ 1 & 1 & 591 & 88.04\end{array}$

JOPJ01000020 Acetobacter okinawensis NC_007512 Pelodictyon luteolum

NZ_FNAM01000033 Sporomusa acidovorans NEVA01000012 Opitutae bacterium

NZ_CVTD020000007 Herbinix hemicellulosilytica AB510668 Bacteroides barnesiae

AJLS01000008 Bacillus bataviensis NEVA01000012 Opitutae bacterium NC_013523 Sphaerobacter thermophilus NC_013523 Sphaerobacter thermophilus NZ_MVHN01000013 Mycobacterium celeriflavum NZ_AAVN02000003 Collinsella aerofaciens NZ_JTDA01000018 Coprobacter secundus NZ_AFII01000001 Parvimonas sp.

NC_003869 Caldanaerobacter subterraneus HE575173 Weissella thailandensis ASTA01000016 Anaerotruncus sp. AJLS01000008 Bacillus bataviensis NZ_NFHC01000009 Flavonifractor sp. NC_013523 Sphaerobacter thermophilus NZ_FNID01000014 Acetanaerobacterium elongatum NC_013523 Sphaerobacter thermophilus NFIX01000011 Muribaculum sp. NZ_CAEG01000015 Alistipes sp. NEUK01000069 Spartobacteria bacterium NSIL01000016 Acidobacterium sp. CP030257 Malassezia restricta NFIX01000011 Muribaculum sp. NZ_KZ614145 Rummeliibacillus pycnus 


\begin{tabular}{|c|c|c|c|c|c|c|c|c|c|}
\hline c155_g1_i1 & 0 & 0 & 0 & 0 & 0 & 13 & 578 & 80.72 & AY017380 Fibrobacter succinogenes \\
\hline c2424_g1_i1 & 0 & 13 & 0 & 0 & 0 & 0 & 603 & 79.59 & AY691296 Ruminococcus flavefaciens \\
\hline c2968_g1_i1 & 0 & 0 & 13 & 0 & 0 & 0 & 595 & 82.51 & CP000386 Rubrobacter xylanophilus \\
\hline c1744_g2_i2 & 0 & 5 & 4 & 0 & 0 & 2 & 591 & 81.25 & NZ_KB894541 Alistipes onderdonkii \\
\hline c281_g1_i1 & 0 & 9 & 0 & 0 & 0 & 0 & 594 & 87.84 & NZ_AUMP01000054 Fictibacillus gelatini \\
\hline c2284_g1_i1 & 5 & 0 & 0 & 1 & 0 & 2 & 577 & 93.3 & AF237462 Streptococcus oralis \\
\hline c2156_g1_i1 & 0 & 5 & 0 & 0 & 0 & 0 & 598 & 99.64 & AF245673 Lactococcus lactis \\
\hline c2425_g1_i1 & 0 & 0 & 0 & 0 & 0 & 4 & 586 & 83.33 & NZ_CVTD020000007 Herbinix hemicellulosilytica \\
\hline
\end{tabular}


References

Abram, P. K., Guerra-Grenier, E., Després-Einspenner, M.-L., Ito, S., Wakamatsu, K., Boivin, G. and Brodeur, J. (2015). An Insect with Selective Control of Egg Coloration. Curr. Biol. 25, 20072011.

Alkhedir, H., Karlovsky, P. and Vidal, S. (2010). Effect of light intensity on colour morph formation and performance of the grain aphid Sitobion avenae F. (Homoptera: Aphididae). J. Insect Physiol. 56, 1999-2005.

Ankersmit, G. and Dijkman, H. (1983). Alatae production in the cereal aphid Sitobion avenae. Neth. J. Plant Pathol. 89, 105-112.

Ankersmit, G., Acreman, T. and Dijkman, H. (1981). Parasitism of colour forms in Sitobion avenae. Entomol. Exp. Appl. 29, 362-363.

Araya, J. E., Cambron, S. E. and Ratcuffe, R. H. (1996). Development and Reproduction of Two Color Forms of English Grain Aphid (Homoptera: Aphididae). Environ. Entomol. 25, 366-369.

Barton, K. and Barton, M. K. (2019). Package 'MuMIn.' R Package Version 1,.

Bates, D., Maechler, M., Bolker, B., Walker, S., Christensen, R. H. B., Singmann, H., Dai, B., Scheipl, F., Grothendieck, G. and Green, P. (2018). Package 'Ime4.' Version 1, 17.

Bowie, J., Cameron, D., Findlay, J. and Quartey, J. (1966). Haemolymph pigments of aphids. Nature 210, 395-397.

Braendle, C. and Weisser, W. W. (2001). Variation in escape behavior of red and green clones of the pea aphid. J. Insect Behav. 14, 497-509.

Caillaud, M. C. and Losey, J. E. (2010). Genetics of color polymorphism in the pea aphid, Acyrthosiphon pisum. J. Insect Sci. 10, 95.

Chroston, J. (1983). Colour polymorphism of the English grain aphid (Sitobion avenae F.).

Cloudsley-Thompson, J. L. (1999). Multiple factors in the evolution of animal coloration. Naturwissenschaften 86, 123-132.

Cott, H. B. (1940). Adaptive coloration in animals.

Dion, E., Polin, S. E., Simon, J.-C. and Outreman, Y. (2011). Symbiont infection affects aphid defensive behaviours. Biol. Lett. 7, 743-746.

Dixon, A. F. G. (1972). Control and Significance of the Seasonal Development of Colour Forms in the Sycamore Aphid, Drepanosiphum platanoides (Schr.). J. Anim. Ecol. 41, 689-697.

Dixon, A. F. G. (1985). Aphid Ecology An optimization approach. Dordrecht: Springer Netherlands.

Dixon, A. F. G. and Agarwala, B. K. (1999). Ladybird-induced life-history changes in aphids. Proc. $R$. Soc. B Biol. Sci. 266, 1549-1553. 
Douglas, A. (1998). Nutritional interactions in insect-microbial symbioses: aphids and their symbiotic bacteria Buchnera. Annu. Rev. Entomol. 43, 17-37.

Duarte, R. C., Flores, A. A. and Stevens, M. (2017). Camouflage through colour change: mechanisms, adaptive value and ecological significance. Philos. Trans. R. Soc. B Biol. Sci. 372, 20160342.

Ford, E. B. (1966). Genetic polymorphism. Proc. R. Soc. Lond. B Biol. Sci. 164, 350-361.

Forsman, A., Ahnesjö, J., Caesar, S. and Karlsson, M. (2008). A model of ecological and evolutionary consequences of color polymorphism. Ecology 89, 34-40.

Fox, J. and Weisberg, H. S. (2011). An R Companion to Applied Regression. 2nd ed. Sage, Thousand Oaks, CA, USA.

Frédérich, B., Mills, S., Denoël, M., Parmentier, E., Brié, C., Santos, R., Waqalevu, V. and Lecchini, D. (2010). Colour differentiation in a coral reef fish throughout ontogeny: habitat background and flexibility. Aquat. Biol. 9, 271-277.

Gallo-Franco, J. J., Duque-Gamboa, D. N. and Toro-Perea, N. (2019). Bacterial communities of Aphis gossypii and Myzus persicae (Hemiptera: Aphididae) from pepper crops (Capsicum sp.). Sci. Rep. 9, 1-12.

Green, S. D., Duarte, R. C., Kellett, E., Alagaratnam, N. and Stevens, M. (2019). Colour change and behavioural choice facilitate chameleon prawn camouflage against different seaweed backgrounds. Commun. Biol. 2, 1-10.

Grigorescu, A. S., Renoz, F., Sabri, A., Foray, V., Hance, T. and Thonart, P. (2018). Accessing the hidden microbial diversity of aphids: an illustration of how culture-dependent methods can be used to decipher the insect microbiota. Microb. Ecol. 75, 1035-1048.

Han, D., Xie, S., Lei, W., Zhu, X. and Yang, Y. (2005). Effect of light intensity on growth, survival and skin color of juvenile Chinese longsnout catfish (Leiocassis longirostris Günther). Aquaculture 248, 299-306.

Hand, S. C. and Wratten, S. D. (1985). Production of sexual morphs by the monoecious cereal aphid Sitobion avenae. Entomol. Exp. Appl. 38, 239-247.

Harmon, J. P., Losey, J. E. and Ives, A. R. (1998). The role of vision and color in the close proximity foraging behavior of four coccinellid species. Oecologia 115, 287-292.

Hazel, W. N. (2002). The environmental and genetic control of seasonal polyphenism in larval color and its adaptive significance in a swallowtail butterfly. Evolution 56, 342-348.

Hebert, P. D., Cywinska, A., Ball, S. L. and Dewaard, J. R. (2003). Biological identifications through DNA barcodes. Proc. R. Soc. Lond. B Biol. Sci. 270, 313-321.

Hille Ris Lambers, D. (1966). Polymorphism in aphididae. Annu. Rev. Entomol. 11, 47-78.

Hougardy, E. and Mills, N. J. (2008). Comparative life history and parasitism of a new colour morph of the walnut aphid in California. Agric. For. Entomol. 10, 137-146. 
Hu, Z.-Q., Zhao, H.-Y. and Thieme, T. (2013). The Effects of Enhanced Ultraviolet-B Radiation on the Biology of Green and Brown Morphs of Sitobion avenae (Hemiptera: Aphididae). Environ. Entomol. 42, 578-585.

Jenkins, R. L., Loxdale, H. D., Brookes, C. P. and Dixon, A. F. G. (1999). The major carotenoid pigments of the grain aphid Sitobion avenae (F.) (Hemiptera: Aphididae). Physiol. Entomol. 24, 171-178.

Josef, N., Amodio, P., Fiorito, G. and Shashar, N. (2012). Camouflaging in a complex environmentoctopuses use specific features of their surroundings for background matching. PLoS One 7, e37579.

Kayser, H. (1982). Carotenoids in insects. In Carotenoid chemistry and biochemistry, pp. 195-210. Elsevier.

Langmead, B. and Salzberg, S. L. (2012). Fast gapped-read alignment with Bowtie 2. Nat. Methods 9, 357.

Libbrecht, R., Gwynn, D. M. and Fellowes, M. D. E. (2007). Aphidius ervi Preferentially Attacks the Green Morph of the Pea Aphid, Acyrthosiphon pisum. J. Insect Behav. 20, 25-32.

Lin, Q., Lin, J. and Huang, L. (2009). Effects of substrate color, light intensity and temperature on survival and skin color change of juvenile seahorses, Hippocampus erectus Perry, 1810. Aquaculture 298, 157-161.

Links, M. G., Chaban, B., Hemmingsen, S. M., Muirhead, K. and Hill, J. E. (2013). mPUMA: a computational approach to microbiota analysis by de novo assembly of operational taxonomic units based on protein-coding barcode sequences. Microbiome 1, 23.

Losey, J. E., Ives, A. R., Harmon, J. P., Ballantyne, F. and Brown, C. (1997). A polymorphism maintained by opposite patterns of parasitism and predation. Nature 388, 269-272.

Loxdale, H. D. and Lushai, G. (2003). Rapid changes in clonal lines: the death of a 'sacred cow.' Biol. J. Linn. Soc. 79, 3-16.

Lymbery, A. (1992). The environmental control of colouration in a bushcricket, Mygalopsis marki Bailey (Orthoptera: Tettigoniidae). Biol. J. Linn. Soc. 45, 71-89.

Majerus, M. (1998). Melanism: evolution in action. Oxford University Press Oxford.

Markkula, M. and Rautapää, J. (1967). The effect of light and temperature on the colour of the English grain aphid, Macrosiphum avenae (F.)(Hom., Aphididae). Societas Entomologica Fennica.

Merilaita, S. and Stevens, M. (2011). Crypsis through background matching. Anim. Camoufl. Mech. Funct. 17-33.

Moran, N. A. and Jarvik, T. (2010). Lateral Transfer of Genes from Fungi Underlies Carotenoid Production in Aphids. Science 328, 624-627. 
Moss, R., Jackson, R. R. and Pollard, S. D. (2006). Mask of wax: secretions of wax conceal aphids from detection by spider's eyes. N. Z. J. Zool. 33, 215-220.

Noor, M. A., Parnell, R. S. and Grant, B. S. (2008). A reversible color polyphenism in American peppered moth (Biston betularia cognataria) caterpillars. PloS One 3, e3142.

Oksanen, J., Blanchet, F. G., Kindt, R., Legendre, P., Minchin, P. R., O'Hara, R. B., Simpson, G. L., Solymos, P., Henry, H., Wagner, S., et al. (2015). vegan: Community Ecology Package. $R$ package version 2.3-0. http://CRAN.R-project.org/package=vegan .

Oliver, K. M., Smith, A. H. and Russell, J. A. (2014). Defensive symbiosis in the real world - advancing ecological studies of heritable, protective bacteria in aphids and beyond. Funct. Ecol. 28, 341-355.

Pérez-López, E., Olivier, C. Y., Luna-Rodríguez, M., Rodríguez, Y., Iglesias, L. G., Castro-Luna, A., Adame-García, J. and Dumonceaux, T. J. (2016). Maize bushy stunt phytoplasma affects native corn at high elevations in Southeast Mexico. Eur. J. Plant Pathol. 145, 963-971.

Polin, S., Le Gallic, J.-F., Simon, J.-C., Tsuchida, T. and Outreman, Y. (2015). Conditional Reduction of Predation Risk Associated with a Facultative Symbiont in an Insect. PLOS ONE 10, e0143728.

R Core Team (2019). $R$ : A language and environment for statistical computing. $R$ Foundation for Statistical Computing, Vienna, Austria.

Roff, D. and Fairbairn, D. (2013). The costs of being dark: the genetic basis of melanism and its association with fitness-related traits in the sand cricket. J. Evol. Biol. 26, 1406-1416.

Saunders, D. S. (2012). Insect photoperiodism: seeing the light. Physiol. Entomol. 37, 207-218.

Schaefer, H. M. and Stobbe, N. (2006). Disruptive coloration provides camouflage independent of background matching. Proc. R. Soc. B Biol. Sci. 273, 2427-2432.

Shamim, G., Ranjan, S. K., Pandey, D. M. and Ramani, R. (2014). Biochemistry and biosynthesis of insect pigments. Eur. J. Entomol. 111, 149-164.

Shu-Sheng, L. and Carver, M. (1982). The effect of temperature on the adult integumental coloration of Aphidius smithi. Entomol. Exp. Appl. 32, 54-60.

Smithers, S. P., Rooney, R., Wilson, A. and Stevens, M. (2018). Rock pool fish use a combination of colour change and substrate choice to improve camouflage. Anim. Behav. 144, 53-65.

Stegen, J. C., Gienger, C. and Sun, L. (2004). The control of color change in the Pacific tree frog, Hyla regilla. Can. J. Zool. 82, 889-896.

Stevens, M. and Merilaita, S. (2009). Animal camouflage: current issues and new perspectives. Philos. Trans. R. Soc. B Biol. Sci. 364, 423-427.

Stoehr, A. M. (2006). Costly melanin ornaments: the importance of taxon? Funct. Ecol. 20, 276-281.

Stringham, G. R. (1971). Genetics of four hypocotyl mutants in Brassica campestris L. J. Hered. 62, 248-250. 
Sullivan, D. J. (2008). Aphids (Hemiptera: Aphididae). In Encyclopedia of Entomology (ed. Capinera, J. L.), pp. 191-215. Dordrecht: Springer Netherlands.

Sutherland, O. R. W. (1969). The role of crowding in the production of winged forms by two strains of the pea aphid, Acyrthosiphon pisum. J. Insect Physiol. 15, 1385-1410.

Tanaka, S. (2004). Environmental Control of Body-Color Polyphenism in the American Grasshopper, Schistocerca americana. Ann. Entomol. Soc. Am. 97, 293-301.

Tanaka, S., Harano, K., Nishide, Y. and Sugahara, R. (2016). The mechanism controlling phenotypic plasticity of body color in the desert locust: some recent progress. Curr. Opin. Insect Sci. 17, 10-15.

Thery, M. and Gomez, D. (2010). Insect colours and visual appearance in the eyes of their predators. In Advances in insect physiology, pp. 267-353. Elsevier.

True, J. R. (2003). Insect melanism: the molecules matter. Trends Ecol. Evol. 18, 640-647.

Tsuchida, T., Ryuichi, K., Horikawa, M., Tsunoda, T., Maoka, T., Matsumoto, S., Simon, J.-C. and Fukatsu, T. (2010). Symbiotic Bacterium modifies aphid body color. Science 330, 109-111.

Van Baaren, J., Wist, T., Soroka, J. and Tougeron, K. (2019). Host-parasitoid network in extreme conditions: the case of cereal aphids in wheat crops in Saskatchewan, Canada. Entomol. Gen.

Watanabe, S., Murakami, T., Yoshimura, J. and Hasegawa, E. (2016). Color polymorphism in an aphid is maintained by attending ants. Sci. Adv. 2, e1600606-e1600606.

Weber, G. (1985). On the ecological genetics of Sitobion a venae (F.)(Hemiptera, Aphididae). J. Appl. Entomol. 100, 100-110. 